\title{
Strukturelle Dialogizität zwischen Experten und Laien: Ideal und Wirklichkeit
}

Zusammenfassung: In der sogenannten Wissensgesellschaft wird die Ressource Wissen als zentraler Faktor für individuelle Entwicklung und gesellschaftlichen Fortschritt proklamiert. Nichtgeteiltes Wissen ist in der Wissensgesellschaft zugleich eine Voraussetzung und Folge der hochspezialisierten Arbeitsteilung und Komplexität der Welt. Die Untersuchung sprachlich gebundener Wissensvermittlung zwischen Fach- und Laienschaft ist politisch und gesellschaftlich hoch relevant, da Bürgerpartizipation in der Wissensgesellschaft untrennbar mit kommunikativen Kompetenzen verbunden ist. Vor diesem Hintergrund wird die strukturelle Dialogizität zwischen Experten und Laien als Denkfigur mit Orientierungsfunktion für partizipative Wissensgesellschaften gesehen. Es stellt sich die grundlegende Frage, wie Wissen unter Berücksichtigung des Aushandlungsdiskurses entsteht und wie die Faktizitätsherstellung für die Akteure und Rezipienten von Laien-Experten-Interaktionen transparent gemacht werden kann. Wissen ist grundsätzlich nicht nur abhängig vom Erkenntnisgegenstand, sondern durch das erkennende Subjekt geformt - und zwar zeichengebunden, vorläufig sowie perspektiven- und interessengeleitet: Wissen zeigt sich im öffentlichen Diskurs stets im Spannungsfeld von Daten und Fakten. Der Beitrag stellt ein Modell idealtypischer Transformationen im Vermittlungsprozess vor und unterscheidet hierbei fünf Vermittlungsmodi. Er verdeutlicht damit die linguistischen Herausforderungen in der Vermittlungsforschung und die Politizität (Relevanz für politisches Handeln) der Linguistik als deskriptiv orientierte Wissenschaft.

Abstract: In the so-called society of knowledge, knowledge as a resource has been claimed to be a vital component for individual development and social progress. At the same time, knowledge which is not shared is both condition for and the result of a highly specialised division of labour and a complex world. The analysis of linguistic knowledge transfer between professionals and the general public is of considerable social relevance since civic participation is

Anmerkung: Für wertvolle und weiterführende Hinweise danke ich Hanna Strub.

Felder, Ekkehard: Universität Heidelberg, Germanistisches Seminar, Hauptstraße 207-209, 69117 Heidelberg, ekkehard.felder[at]gs.uni-heidelberg.de 
intrinsically tied to communicative competences in a society of knowledge. Due to these conditions, the establishment of a structural dialogue between experts and non-experts is seen as an idea which participative societies of knowledge may use as a reference point. The underlying question is how knowledge generated (specifically when regarding the negotiations within the discourse) and how the creation of facts can be made transparent for actors and recipients of interactions between experts and non-experts. In general, knowledge is not only dependant on the matter at hand but also formed by the perceiving subject; it is bound to symbols, preliminary and influenced by perspectives and interests. In public discourse we perceive knowledge in relation to data and facts. This paper presents a model of typical transformations within the mediation process, distinguishing between five modes of mediation. It shows the linguistic challenges of mediation studies and the political relevance of linguistics as a descriptive field.

Schlüsselwörter: agonale Zentren, Daten, Dialogizität, Experten-LaienKommunikation, Fakten, Faktengenese, Vermittlung, Vermittlungsmodi, Wissen, Wissensgesellschaft

\section{Einleitung}

Um es gleich vorweg zu sagen: Sprachwissenschaftliche Analysen von Wissensbeständen und die Untersuchung der sprachlich gebundenen Wissensvermittlung sind politisch und gesellschaftlich von höchstem Nutzen. Mit den folgenden Ausführungen will ich en passant belegen, wie politisch bzw. von welcher politischen Relevanz sprachwissenschaftliche Forschung in ihrem deskriptiven Duktus sein kann. Politisch meine ich nicht in einem parteipolitischen Sinne, sondern in einem gesellschaftspolitischen. Während landauf und landab darüber nachgedacht wird, wie die teilweise vergiftete Atmosphäre zwischen sog. Elite und denjenigen, die sich nicht zu dieser Gruppe zählen, verbessert werden kann, können Sprachwissenschaftler auf eine gewisse Forschungstradition im Bereich Vermittlung blicken. Manche nennen diesen Bereich auch Wissenstransfer bzw. Transferwissenschaft oder Experten-Laien-Kommunikation, aber das vergebene Etikett ist zweitrangig (vgl. zur linguistischen Analyse des Wissenstransfers Antos \& Wichter 2005 und Wichter \& Antos 2001).

Ich bevorzuge die Vokabel „Vermittlung“ (Steger 1988: 314; Becker 2001), weil sie zwischen Fachlichkeit und Laienschaft eine - quasi vor dem geistigen Auge entstehende - Brücke schlägt und auch nicht kaschiert, dass Experten 
gegenüber Laien einen Wissensvorsprung haben - der selbstredend auch immer interessengeleitet ist. Man kann sogar von einer systematischen Wissensasymmetrie sprechen. Zudem bevorzuge ich den Ausdruck Vermittlung, weil Vermittlung ein einfach verstehbares deutschsprachiges Wort ist.

Die Relevanz von Vermittlungsfragen und -aspekten wird von Experten wie Laien gleichermaßen als wichtig eingeschätzt. Das ist erfreulich. Dieser Gesichtspunkt ist nicht trivial - weil auch Wissenschaft als Ganzes nicht mehr per se als sinnvoll und notwendig erachtet wird, sondern - auch oder gerade durch den besonderen Status der Wissenschaft im Kontext der Corona-Pandemie ab 2020 - ebenfalls um Akzeptanz werben muss. Mit dieser Position, dass also Wissenschaft sich um Vermittlung bemühen muss (daher war der Transfergedanke im letzten Exzellenz-Strategiewettbewerb der deutschen Universitäten von fundamentaler Bedeutung), geht aber auch eine Erwartungshaltung einher. Erwartungen können leider auch enttäuscht werden, also müssen wir uns anstrengen und genau überlegen, was wir versprechen. Deswegen lautet dieser Beitrag im Untertitel „Ideal und Wirklichkeit“.

\section{Gesellschaftliche Relevanz sprachwissenschaftlicher Expertise in der Experten-Laien-Kommunikation}

Dass wir in einer sog. Wissensgesellschaft leben ${ }^{1}$ und diese in besonderem Maße auf kommunikative Kompetenzen seiner Bürger angewiesen ist (vgl. Felder 2006; Keller 2010; Knoblauch 1995), stellt einen Gemeinplatz dar. Daraus folgt: Wer an der Ressource Wissen partizipieren will, muss kommunizieren können was die Rezeption und Produktion kommunikativer Interaktionen anbelangt. Ohne Sprachkompetenz keine Bürgerpartizipation. Diese Selbstverständlichkeit hat in den letzten Jahren im Kontext der Debatte um Populismus in der Politik eine neue Dynamik erfahren. Viel ist von einer Blase die Rede, in denen sich tatsächlich oder vermeintlich die Eliten bewegen oder diejenigen, die sich selbst als ,das Volk' bezeichnen.

All dies ist hier insofern relevant, als die Ressource Wissen und der Umgang mit ihr die zentrale Voraussetzung für gesellschaftliche Teilhabe, aber vor allem

1 Vgl. zur Programmatik, Begriffskritik und zum theoretischen Hintergrund z. B. Bittlingmayer \& Bauer 2006; Engelhardt \& Kajetzke 2015; Weingart, Carrier \& Krohn 2007. 
auch für wirtschaftlichen Erfolg darstellt. Und da Wissenserwerb sprachlich und kommunikativ gebunden ist, so ist die Linguistik zuständig. Vor diesem Hintergrund will ich im Folgenden die Denkfigur der strukturellen Dialogizität (vgl. Felder 2018a: 236) als Orientierungskonzept (handlungsleitendes Konzept) in den Mittelpunkt rücken. Dies geschieht vor dem Hintergrund, dass Wissensvermittlung früher (und zuweilen heute noch) als ein monologischer Akt gesehen wurde - der mitunter wunderliche Blüten treibt und sich in ironischer Weise idiomatisiert hat in dem phrasenhaften Redeeinstieg Pass mal auf und hör gut $z u$. Die Zeiten des reinen Zuhörens sind aber schon lange vorbei. Zum Stand der Forschung über den Status Experte und Laie sei auf das - in der HSW-Reihe 2019 erschienene „Handbuch im Urteil der Öffentlichkeit“ verwiesen und dort auf den Beitrag von Bock \& Antos (2019). Dort geht es um rollensoziologische Aspekte wie auch Nichtwissen-Typen (vgl. dazu die vielfältigen Facetten in dem von Janich, Nordmann \& Schebek 2012 herausgegebenen Band „Nichtwissenskommunikation in den Wissenschaften“, vgl. auch die Beiträge von Antos; Frase \& Harnisch; Kasper \& Purschke sowie Spitzmüller, alle in diesem Band).

Im Folgenden soll der Blick auf etwas Anderes gerichtet werden: Der Fokus der hier erörterten Vermittlungsproblematik liegt auf den linguistischen Konsequenzen, die sich im Kontext der oben erwähnten Wissensgesellschaft ergeben - nämlich dem sprachlich gebundenen Erwerb von Wissen: Die Gewinnung und Verteilung von Wissen ist derjenige Aspekt des menschlichen Zusammenlebens, welcher die postindustriellen Gesellschaften am prägnantesten charakterisiert. Die Ausdrücke Gewinnung und Verteilung indizieren dabei eine gesellschaftliche Asymmetrie, die als Problem der Wissensgesellschaft vordringlich ist. Um an dieser teilhaben zu können, bedarf es bestimmter Kompetenzen. Die Sicherstellung dieses Kompetenzerwerbs ist unter anderem nicht nur eine bildungspolitische, sondern vor allem auch eine linguistische Aufgabe, wenn kommunikative Partizipation an der Wissensgesellschaft als eine Manifestation sozialer Gerechtigkeit verstanden wird (vgl. zur soziokommunikativen Konstruktion bestimmter Wissensbereiche Knoblauch 1995). Diese soziologisch, politologisch, philologisch und bildungspolitisch inspirierte Sicht auf Gesellschaft als eine - von sozialen und kulturellen Praktiken geprägte - Formation stellt nicht nur eine theoretische Modellierung gesellschaftlichen Handelns dar, sondern sie kann in den interdisziplinären Vermittlungskontexten zu einem Leitgedanken und Grundsatz avancieren.

Diese Gedanken haben Auswirkungen auf die Statuskonzepte von Laie und Experte. Beide gilt es nicht als hermetisch abgeschlossen anzunehmen. Vielmehr ist das Ideal einer dialogischen Grundkonzeption zweier sich gegenseitig beeinflussender Protagonisten-Prototypen zugrunde $\mathrm{zu}$ legen, die zum einen 
interagieren und zum anderen sich in ihren sozialen und kognitiven Rollen (vgl. zu der Unterscheidung zwischen Experte und Laie bei Bromme, Jucks \& Rambow 2004) gegenseitig hinsichtlich Wissensgenese und Kommunikationsform beeinflussen (vgl. dazu wissenssoziologische Aspekte aus linguistischer Sicht bei Becker 2001: 115 und grundständig aus soziologischer Perspektive Keller 2010). Und der Laie ist für das Verstehen mitverantwortlich, muss es nach seinen Möglichkeiten sichern und gegebenenfalls Wissen durch richtige Fragen einfordern (vgl. Heringer 1979, 1984). In diesem Zusammenhang muss daran erinnert werden, dass Laien sich informieren müssen, um Entscheidungen treffen $\mathrm{zu}$ können. Daher ist ein häufig erwähntes Ziel der Experten-LaienKommunikation das Ermöglichen einer informierten Entscheidung (vgl. zur Linguistik des Entscheidens Jacob 2017).

Daraus ergibt sich die Frage, welche gesellschaftlichen Voraussetzungen vonnöten sind, solch ein Ideal der strukturellen Dialogizität (vgl. Felder 2018a: 236) zumindest ansatzweise oder graduell umzusetzen. Diese Aspekte möchte ich nun im Folgenden entfalten.

\section{Strukturelle Dialogizität als Denkfigur mit Orientierungsfunktion für partizipative Wissensgesellschaften}

Strukturelle Dialogizität als Kommunikationsprinzip fokussiert hier die Beziehung zwischen Laien und Experten und betrifft die drei Komponenten des Bandes: Laien - Wissen - Experten. Sie stellt eine Orientierungsfunktion partizipativer Wissensgesellschaften dar und kann eine demokratische Kommunikationskultur stärken. Strukturelle Dialogizität ist eine Denkfigur, die aus der Antike kommt und in der politischen Rhetorik Tradition hat. In der Gegenwart findet sie in Vertretern wie Jürgen Habermas und Josef Kopperschmidt ihre Zuspitzung: Sie beharren auf der „Symmetrie von Berechtigungen und Verpflichtungen“ (Habermas 1971: 138) im Diskurs. Dieser Denkansatz sollte ein Leitbild oder Orientierungspunkt für die Experten-Laien-Kommunikation sein.

Demokratische Kommunikationsstrukturen liegen vor, wenn öffentliche Aushandlung dem Geiste nach auf Partizipation (,Teilhabe`) angelegt ist. Kopperschmidt plädiert unter Bezugnahme auf Habermas für ein Modell der öffentlichen Rede, 
das von der Kommunikations- bzw. Redesituation ausgeht, in der ein Einzelsubjekt als Redner monologisch sein Redeziel zwar zustimmungsfähig zu machen versucht, in der Verständigung [...] aber nur gelingen kann, wenn diese Einzelrede strukturell dialogisch dimensioniert ist, d.h. wenn ihre formale Monologizität durch die gelingende Einbettung in kommunikativ übergreifende Verständigungsprozesse strukturell aufgefangen wird.

(Kopperschmidt 1990: 495)

Auch die Herstellung von Wissen und ihre Distribution können bekanntermaßen umstritten sein (vgl. Felder 2006; Warnke 2009). Streit oder Kämpfe um Geltungsansprüche sind also nicht zu vermeiden, sondern zu kultivieren. Auf die fachexterne Kommunikation übertragen nimmt dieser Denkansatz Experten wie Laien gleichermaßen in die Pflicht und kann ein Orientierungspunkt für die Modellierung eines Vermittlungsdialogs (Experten-Laien-Kommunikation) sein.

Dabei kommt den Experten und Vermittlern besondere Verantwortung zu denn: Wer definiert, regiert (vgl. Schnedermann i. Vorb.: „Die Macht des Definierens“). Wer Wissen sprachlich konstituiert, der instruiert das Denken. Damit einher geht eine gesamtgesellschaftliche Verantwortung - vor allem dem Laien gegenüber. Denn Herrschaft und Macht werden auch über sprachliche Zeichen und ihre Verwendung ausgeübt. Betrachtet man Sprache als Medium zur Durchsetzung bestimmter Sichtweisen auf gesamtgesellschaftlich relevante Sachverhalte in unterschiedlichen Wissensdomänen (vgl. die Beiträge in dem Sammelband „Semantische Kämpfe“ (Felder 2006) zu Medizin, Wirtschaft, Architektur, Naturwissenschaft, Technik, Politik, Geschichte, Recht usw. und die von Felder \& Gardt (2015-2020) herausgegebene Reihe „Handbücher Sprachwissen“), so offenbaren sich ,hinter' fachlichen Auseinandersetzungen gleichsam Auseinandersetzungen um angemessene Bezeichnungen, um Bedeutungszuschreibungen und um die sprachliche Passung bzw. Fixierung abstrakter Sachverhalte (Dimensionen im Paradigma des Semantischen Kampfes). Wir haben es mit einer Auseinandersetzung um Definitionshoheit zum Zwecke der perspektivierten Wissenskonstitution (gesellschaftlichen Einflussnahme) zu tun - kurzum mit semantischen Kämpfen (vgl. Felder 2006) oder semantischen Wettkämpfern².

Dadurch macht sich der gesellschaftliche Diskurs abhängig - und zwar von den ,Definierern'. Denn aus linguistischer Sicht zeigt sich, wie Sprache, vor' der Konstituierung der Sachverhalte die fachspezifischen Wissensrahmen (mit)strukturiert, wie also Wissen durch Sprache entsteht. Aus diesen Befunden resultieren Anforderungen an Laien (was die Verstehbarkeit der komplexen

2 So der Titel meines Blogs auf https://scilogs.spektrum.de/semantische-wettkaempfe/ (letzter Zugriff 06.07.2020). 
Fachzusammenhänge anbelangt) und Experten (was das Transparenz-Machen der Wissensgenese betrifft), die es in sich haben. Anspruch der Verstehbarkeit und des Transparenz-Machens einerseits und Wirklichkeit andererseits sind kaum in Übereinstimmung zu bringen.

Gesellschaftspolitisch ist diese Diagnose solange kein Problem, wie es Gegenentwürfe gibt - kurz ,Gegendefinierer‘. Perspektivenvielfalt und Multiperspektivität sind Stabilitätsgaranten für demokratische Gesellschaften, wenn sie keinem beliebigen Relativismus anheimfallen und (gemäß dem Grice'schen Kooperationsprinzip) dem Wahrhaftigkeitsprinzip verpflichtet fühlen. Multiperspektivität und die Durchsetzung von Wahrheitsansprüchen schließen sich nicht aus, sondern ersteres ist die Voraussetzung für letzteres - wenn man von Folgendem ausgeht:

Behauptungen mit standpunkttranszendenten Wahrheitsansprüchen sind obsolet, assertorische Aussagen mit objektiviertem Wahrheits- und Gültigkeitsanspruch sind von höchster Relevanz. Diese Aussageformen sind das Schmiermittel demokratischer Diskurse im Paradigma der strukturellen Dialogizität.

(Felder 2018b: 392)

Daraus folgt: Die perspektivierte Wissenskonstitution gesellschaftlicher Diskursakteure ist im Vermittlungsprozess so weit wie möglich sichtbar zu machen - (Wett-) Kämpfe um Gültigkeitsansprüche von fachlichen Darstellungen sind also nicht zu vermeiden, sondern für Laien luzide zu kultivieren.

\section{Daten-Fakten-Unterscheidung als Heuristik zum Transparent-Machen der interessengeleiteten Faktengenese}

Im vorliegenden Ansatz ist zu klären, wie die Konstituierung von Wissensbeständen adäquat zu beschreiben ist, damit diese Erkenntnisse für die Vermittlung von Wissen fruchtbar gemacht werden können. Es ist dabei zu reflektieren, wie bei der vermittlungs-, fach- und gemeinsprachlichen Wirklichkeitskonstitution aus Daten (als unstrittiger Diskursinput) Fakten (Aussagen bzw. Propositionen, über deren Wahrheitsgehalt gestritten werden kann) generiert werden. 


\subsection{Wer Daten verarbeitet, macht Fakten: Fakten als Interventionsmittel}

Die oben dargelegten Gesichtspunkte verdichten sich in der grundlegenden Frage, wie Wissen entsteht und wie die Faktizitätsherstellung für die Akteure und Rezipienten von Laien-Experten-Interaktionen transparent gemacht werden kann. Beim Anführen von Daten (als uninterpretierte Menge an unstrittigen Wissenseinheiten bzw. Informationen) und dem Herstellen von Fakten (als Aussagen, um deren Geltung gestritten werden kann) sind verschiedene Aspekte zu unterscheiden (vgl. dazu Felder 2013: 14; Felder 2018b: 384-386). Im Folgenden soll die Unterscheidung von Daten und Fakten fruchtbar gemacht werden, die Darstellungen resümieren die Darlegungen in Felder (2013).

\subsection{Von Tatsachen, Daten und Fakten als Interventionsbeitrag}

Ausgangspunkt der terminologischen Überlegungen ist das Wort Tatsache, das menschliches Tun (Tat-) und ontisch gegebene Entitäten (-sache) vereint. Der Ausdruck ist laut „Duden - Das Herkunftswörterbuch“ (vgl. Riecke 2014) im 18. Jahrhundert durch Inspiration des englischen matter of fact aufgekommen, das wiederum auf die lateinische Bezeichnung res facti rekurriert. Soziale Tatsachen bilden dabei einen Sonderfall, weil sie ausschließlich per Übereinkunft akzeptiert und dadurch sukzessive konventionalisiert werden - sie werden als gesellschaftlich akzeptierte Wirklichkeit konstituiert (vgl. Searle 1997). Wissen als Orientierungsgröße menschlichen Verhaltens und Denkens ist auf zweierlei angewiesen: auf Unumstößliches und auf daraus gewonnene Schlussfolgerungen.

Aus diesem Grund sind die beiden Termini Daten und Fakten (als die beiden zentralen Komponenten des Wissensbegriffs) voneinander abzugrenzen und eine synonyme Verwendung möglichst zu vermeiden, obwohl gerade eine solche Synonymität (beide Ausdrücke stehen für Unumstößliches) sich schon seit einiger Zeit im Sprachgebrauch auszubreiten scheint (vgl. Felder 2013: 14). Ein Blick auf die Infinitive facere (lat. `machen`) und dare (lat. sgeben`), die den Verbalabstrakta Faktum (lat. >Gemachtes`) und Datum (lat. >Gegebenes`) zugrunde liegen, stellt Wissen in den epistemologisch schwierigen Zusammenhang von (Vor)Gegebenem (Daten) und Gemachtem (Fakten). Damit wird zugleich deutlich, dass Wissen einerseits aus intersubjektiv unstrittig Gegebenem besteht - also aus Daten als nach allgemein akzeptierten Kriterien gewonnenen, oft gemessenen Größen. Andererseits basiert Wissen ebenfalls auf Gedeutetem - also auf beobachteten Ereignissen sowie anschließend abstrahierten 
und damit hergestellten Tatsachen als Fakten mit breitem Gültigkeitsanspruch. Streng genommen existieren Daten nur dann, wenn intersubjektiv diese von allen Menschen als existent anerkannt werden, also auf der Basis nachvollziehbarer Intersubjektivität oder Fakten (z. B. Akzeptanz von Himmelsrichtungen als die Richtung von einem Bezugspunkt (z. B. Standort) zu einem anderen Punkt auf der Erdoberfläche).

Heuristisch ist die folgende Trennung nützlich: Fakten sind von Diskursakteuren sinnvoll Gemachtes und von hoher und breit akzeptierter Plausibilität, die - dessen ungeachtet - aber dennoch von anderen Diskursbeteiligten bestritten werden können (z. B. Kriege sind die Hauptursache für die gestiegene Zahl der Flüchtlinge im Jahre 2015 oder Wirtschaftswachstum trägt zum Wohlstand der Menschen bei). Daten dahingegen sind unstrittig, also allseits akzeptiert (z. B. Ein Atom besteht aus einer Hülle und einem Kern, Im Jahr 2014 wurden in der Bundesrepublik Deutschland laut Bundesamt für Migration und Flüchtlinge 202.834 Asylanträge gestellt, Die Bundesrepublik Deutschland gliedert sich in 16 Bundesländer oder Das Phänomen des Stalking („Nachstellung“) soll rechtlich nach dem Willen des Gesetzgebers seit 2007 durch $§ 238$ StGB geregelt werden oder Paris ist die Hauptstadt Frankreichs) (vgl. Felder 2013: 14).

Der diskurslinguistische Beitrag im Tableau gesellschaftspolitisch inspirierter Diskuranalysen besteht darin, Kontroversen in Diskursen darauf zurückzuführen, welche Aussagen (Propositionen) als unstrittige Daten im Diskurs allgemein akzeptiert werden und welche Faktizitätsherstellungen (Fakten) umstritten sind (zu Agonalität im Diskurs vgl. Felder 2013; Mattfeldt 2018). Eine Formulierung wie Paris ist die Hauptstadt Frankreichs dürfte als intersubjektiv unstrittig gelten, während ein als Faktum etikettierter Satz wie Wirtschaftswachstum trägt zum Wohlstand der Menschen bei in dieser allgemeinen Form Kontroversen auslösen dürfte.

Der im Duktus von Objektivität konventionalisierte Sprachgebrauch des Lexems Fakt oder Faktum zeigt sich eindrücklich bei dem in manchen Medienformaten propagierten sogenannten Faktencheck (z. B. in der Fernsehtalkshow hart aber fair). Man könnte sagen: Ein ,Faktencheck' trägt zur Durchleuchtung, nicht aber zur Lösung der Streitfrage bei, ob eine bestimme Aussage (Proposition) intersubjektiv als gültig klassifiziert werden kann (vgl. dazu die Beispiele in Bloching 2018). Der Anspruch der ,Faktizitätsüberprüfung‘ (kann also eine Aussage als Fakt bezeichnet werden und sind die entsprechenden Überprüfungen möglich?) ist schwierig, aber nicht unmöglich. Selbst der Umstand - und das ist mir besonders wichtig -, dass sich zwei streitende Diskursakteure nicht darüber verständigen können, was in ihrem Disput als Datum und was als Faktum zu 
gelten hat, ist für die Rezipienten des Diskurses erkenntnisstiftend (vgl. Bloching 2018).

Und abschließend sei daran erinnert: In diesem Ideal müssten die Wissensgenese und die Charakteristika von Wissensvermittlungsprozessen transparent gemacht werden, dass also Wissen nicht statisch produktorientiert präsentiert wird (als Daten und Fakten), sondern unter prozessualen Aspekten und unter Berücksichtigung des Aushandlungsdiskurses.

\subsection{Beispiel Impfdiskurs und die dort ermittelten agonalen Zentren}

Da in der Regel keine Wissenskonstitution und damit keine Wirklichkeitsperspektive intersubjektiv als einzig gültig akzeptiert wird, können in Weiterführung von Lyotard (1987), Assmann (1999), Felder (2006) und Warnke (2009) in Diskursanalysen agonale Zentren als konfligierende handlungsleitende Konzepte herausgearbeitet werden (vgl. zum pragma-semiotischen Ansatz Felder 2015). Sie stellen widerstreitende Kristallisationspunkte im Diskurs dar. Aus diesem Blickwinkel haben wir es mit einem Wettstreit diskursiv geprägter Weltausschnitte zu tun, die alle um möglichst breite Akzeptanz und Gültigkeit werben (vgl. ganz grundsätzlich Mattfeldt 2018).

Unter agonalen Zentren verstehe ich einen sich in Sprachspielen manifestierenden Wettkampf um strittige Akzeptanz von Ereignisdeutungen, Handlungsoptionen, Geltungsansprüchen, Orientierungswissen und Werten in Gesellschaften. Denn zentrale und umstrittene Grundannahmen - also konfligierende Geltungsansprüche von (Wahrheits-)Aussagen - zu erfassen, steht im Zentrum des diskursanalytischen Untersuchungsprogramms. Im Fokus der Aufmerksamkeit stehen daher kompetitive Sprachspiele zwischen verschiedenen gesellschaftlichen Diskursakteuren. Somit werden gleichsam Diskurse als Orientierungsrahmen geformt und zentrale Variablen in der Aushandlungspraxis je neu justiert und modifiziert. Agonale Zentren haben also nichts Statisches, sondern sind im Gegenteil stets dem diskursiven Aushandlungsprozess ausgesetzt (vgl. Felder 2015: 108). Diese Strittigkeit muss meines Erachtens im Vermittlungsprozess transparent bzw. nachgezeichnet werden.

Agonale Zentren und ihre Ermittlung sind charakterisiert durch konträre handlungsleitende Konzepte und dienen in der Diskursanalyse der folgenden Zielsetzung:

- Der Wettstreit um die angemessene Konzeptualisierung von lebensweltlichen und fachlichen Sachverhalten wird durch die Gegenüberstellung di- 
vergierender Konzepte und konfligierender Geltungsansprüche deutlich, sie heißen agonale Zentren.

- Agonale Zentren in einem Diskurs referieren auf den Wettkampf zur Durchsetzung von Sichtweisen mittels Dominantsetzung bestimmter sprachlicher Mittel.

- Agonale Zentren (= diskursive Wettkämpfe um Geltungsansprüche und adäquate Konzeptualisierungen) legen Diskursstreitigkeiten induktiv aus dem sprachlichen Material offen. Sie verdichten und synthetisieren im Diskurs Impliziertes durch Analyse des Explizierten.

- Die Offenlegung der agonalen Zentren dient unter anderem einem entscheidenden Vermittlungsziel, nämlich der Verdeutlichung konfligierender Konzeptualisierungen im Diskurs. Diese soll der relative Laie (eine Bezeichnung für eine Person, die durch eine aktive Aneignung fachspezifischen Wissens sukzessive kompetenter wird, ohne den Expertenstatus zu erlangen) erfahren.

\section{Ein Beispiel aus dem Impfdiskurs im europäischen Vergleich}

Die Relevanz agonaler Zentren für die Vermittlungsproblematik illustriere ich im Folgenden am Impfdiskurs, der in einem interdisziplinären Projekt des Europäischen Zentrums für Sprachwissenschaften (EZS) in Heidelberg im Mittelpunkt steht. Dort wird in thematischen Korpora des Deutschen, Englischen, Französischen und Italienischen untersucht, wie die Diskussion um das Impfen im europäischen Vergleich geführt wird. Dazu beschäftigt sich die aus verschiedenen Philologien gebildete Projektgruppe im Rahmen des mehrsprachigen Projekts „Europäische Diskursgemeinschaft: Perspektivenfrieden und Perspektivenstreit“" ${ }^{\text {3 }}$ mit Handlungsspezifika von Diskursakteuren. Es sollen sprachliche Mittel an der Sprachoberfläche nachgewiesen werden, die rekurrent vorkommen und mit Typen von Sprachhandlungen korrelieren (vgl. die grundlegende Darstellung des Projektes in Atayan et al. 2020). Dabei interessieren in erster Linie die folgenden Handlungstypen als Kategorien mittlerer Abstraktion (vgl. Felder 2015: 95):

1. Diskursakteure setzen einen Sachverhalt sprachlich fest (Sachverhaltskonstituierung als Sachverhaltsklassifizierung),

$3 \mathrm{http}: / /$ ezs-online.de/de/forschung/gefoerderte-aktivitaeten/europaeischediskursgemeinschaft-perspektivenfrieden-und-perspektivenstreit/ (letzter Zugriff 06.07.2020). 
2. Diskursakteure verorten den festgesetzten Sachverhalt in Relation zu anderen Sachverhalten (Sachverhaltsverknüpfung) und

3. Diskursakteure bewerten den Sachverhalt in der Regel explizit oder implizit (Sachverhaltsbewertung).

Im Mittelpunkt des Erkenntnisinteresses steht die Intention, auf der Basis eines großen thematischen (digitalisierten) Textkorpus (mehrsprachiges Textkorpus) eine linguistische Analysetechnik der Themenkonstitution, -entfaltung und spezifikation (am Exempel des Themas Impfung) zu präsentieren, die sich sowohl hermeneutischer als auch semi-automatisierter Methoden bedient. Im Rahmen der pragma-semiotischen Textarbeit wird der Diskurs auf mehreren sprachwissenschaftlichen Ebenen untersucht, indem - ausgehend vom Diskursthema Impfung - auf der Basis qualitativer und quantitativer Verfahren zentrale Subthemen des Diskurses ermittelt und diese wiederum hinsichtlich der zentralen Grundannahmen innerhalb des Diskurses - also konfligierender Geltungsansprüche von Wahrheitsaussagen - analysiert werden. Solche umstrittenen Grundannahmen werden als agonale Zentren (im Sinne diskursiver Wettkämpfe um Geltungsansprüche) bezeichnet und Praktiken ihrer Ermittlung transparent gemacht. Agonale Zentren werden durch grundlegende und umstrittene handlungsleitende Konzepte (vgl. Felder 2013: 21) modelliert.

An folgendem Beispiel aus der Diskursanalyse soll dies verdeutlicht werden: Es gibt ein unstrittiges Datum im Diskurs, das von allen Diskursakteuren akzeptiert wird: „Die Krankheit der Pocken gilt gegenwärtig in unserem Kulturkreis als ausgerottet.“ Auf der Grundlage dieses Datums wird über die Erklärung bzw. die zentrale Ursache für diesen - von allen Seiten - anerkannten Sachverhalt gestritten und es bilden sich in der Faktengenese divergierende Einschätzungen dazu hinaus. Diese umstrittenen Annahmen stehen sich als divergierende Fakten bzw. handlungsleitende Konzepte gegenüber und legen ein agonales Zentrum offen. Im konkreten Fall stehen sich die beiden handlungsleitenden Konzepte >Die Ausrottung von Krankheiten wie den Pocken liegt an der Impfquote und `Die Ausrottung von Krankheiten wie den Pocken liegt an verbesserten Lebensbedingungen` gegenüber (vgl. Tab. 1). 
Tab. 1: Agonales Zentrum ,Impfen versus Lebensbedingungen`.

\begin{tabular}{|c|c|c|}
\hline Handlungsleitendes Konzept 1 & & Handlungsleitendes Konzept 2 \\
\hline $\begin{array}{l}\text { >Die Ausrottung von Krankheiten wie } \\
\text { den Pocken liegt an der Impfquote.ı }\end{array}$ & VERSUS & $\begin{array}{l}\text { >Die Ausrottung von Krankheiten wie } \\
\text { den Pocken liegt an verbesserten } \\
\text { Lebensbedingungen.؛ }\end{array}$ \\
\hline
\end{tabular}

Die Ermittlung und Offenlegung der agonalen Zentren dient im Vermittlungsprozess zwischen Experten und Laien somit der Vermittlung von unstrittigen Daten wie auch divergenten Fakten aus identischen Daten (vgl. im folgenden Abschnitt 5 die Vermittlungsmodi 4 und 5).

\section{Strukturelle Dialogizität: Modell einer idealtypischen Transformation im Fokus verschiedener Vermittlungsmodi}

Versucht man nun in Bezug auf einen Vermittlungsgegenstand sowohl die systematischen Wissensasymmetrien zwischen Experte und Laie als auch die konvergenten und divergenten Daten und Fakten im Diskurs, die sich bei der Faktengenese ermitteln lassen, im Vermittlungsprozess luzide darzustellen und damit dem Ziel einer partizipativen Kommunikationsgesellschaft gerecht $\mathrm{zu}$ werden, so lassen sich in einem Modell idealtypische Transformationen mittels verschiedener Vermittlungsmodi beschreiben. Dabei sind die folgenden fünf Untergruppen zu unterscheiden:

1. Vollständige Vermittlung des Wissensmoments - d.h. 1:1 Vermittlung (vollständiges Verständnis auf Seiten der Laienperson)

2. Vermittlung von Teilen des Wissensmoments und Transparent-Machen der nicht-vermittelten Inhalte (partielles Verständnis)

3. Vermittlung der Konturen des zu vermittelnden Wissensmoments (intuitives Verständnis)

4. Vermittlung der unstrittigen Inhalte des Wissensmoments (d. h. Daten und Fakten)

5. Vermittlung divergenter Fakten aus identischen und unstrittigen Daten 
Schema
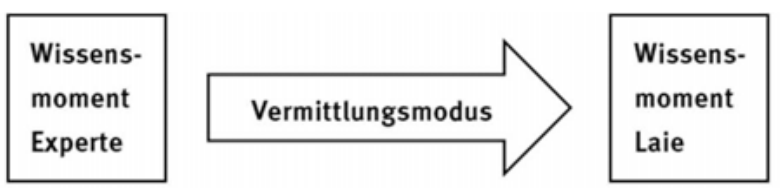

Basis-Modi
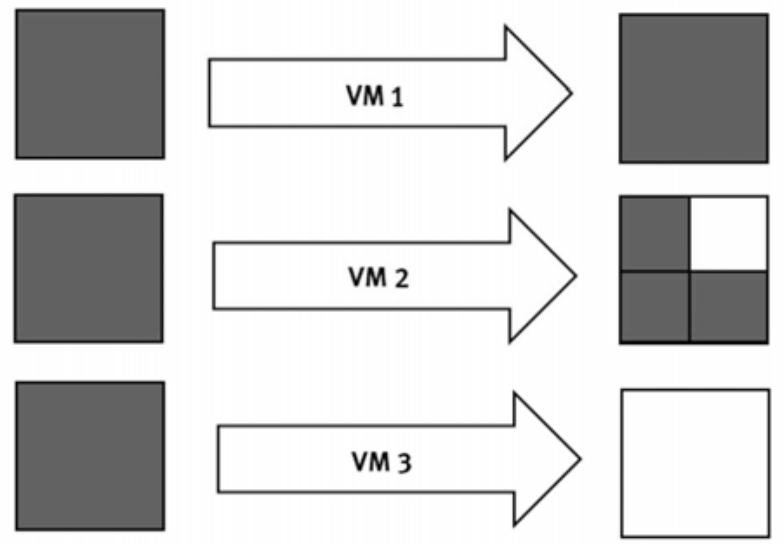

Meta-Modi
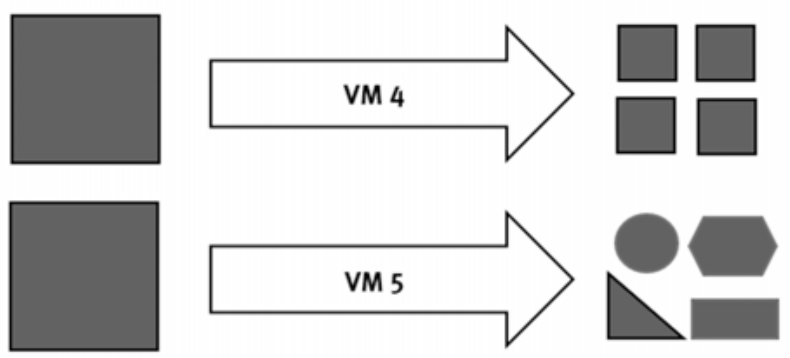

Abb. 1: Modell idealtypischer Vermittlungsmodi (VM) im Vermittlungsprozess zwischen Experten und Laien.

Die Abb. 1 stellt ein Modell der fünf idealtypischen Vermittlungsmodi im Vermittlungsprozess zwischen Experten und Laien dar. Die Vermittlungsmodi 1-3 sind als die drei idealtypischen Basis-Modi der Vermittlung von Wissensmomenten über einen Vermittlungsgegenstand zu verstehen. Die Vermittlungsmodi 4 und 5 sind als Meta-Modi zu denken, da sie über den Inhalt des Vermittlungsgegenstands hinaus die (Un)Strittigkeit der Daten- bzw. Faktenlage vermitteln. Das heißt, dass sich die Vermittlungsmodi 1-3 und die Vermittlungsmodi 4 und 5 auf zwei unterschiedliche Ebenen der Vermittlung beziehen und die Basis-Modi 1-3 mit den Meta-Modi 4 und/oder 5 im Vermittlungsprozess 
kombinierbar sind. Im Folgenden seien die fünf Vermittlungsmodi exemplarisch erläutert:

1. Zunächst ist der Vermittlungsmodus 1 (VM1) anzuführen, der davon ausgeht, dass ein Inhalt 1:1 zu vermitteln ist - gleichsam ohne Komplexitätsreduktion. Ein Beispiel hierfür ist die Vermittlung des Wissensmoments, dass ein menschlicher Körper in der Lage ist, Antikörper als Abwehrreaktion aufzubauen.

2. Bei der Vermittlung des Wissens (VM2) wird die Komplexität transparent reduziert bzw. derart vereinfacht, dass für den Laien erfassbar ist, welche Wissenskomponenten er nicht vollumfänglich zu verstehen in der Lage ist und welche er erfassen kann. Beispielsweise sind die positiven und erwünschten Wirkungen des Impfens erfassbar, negative oder unerwünschte Wirkungen bleiben dagegen nur partiell erfassbar.

3. Vermittlungsmodus 3 (VM3) modelliert die Vermittlung dergestalt, dass der Laie die Wissenskomponenten und ihre Zusammenhänge nur intuitiv erahnen kann. Ein Beispiel hierfür ist der Umgang mit statistischen Größen bei der Einschätzung individueller Gefahrenabwägung zwischen Impfen und Nicht-Impfen.

4. Vermittlungsmodus 4 (VM4) bildet die Vermittlung der unstrittigen Inhalte ab, also zum Beispiel das Datum und Faktum, dass die Ausrottung der Pocken zu begrüßen ist, auch wenn es Streit darüber gibt, durch welche Maßnahmen oder Umstände dieser Zustand herbeigeführt wurde.

5. Vermittlungsmodus 5 (VM5) steht für die Vermittlung divergenter Fakten aus identischen Daten. Ein unstrittiges Datum ist beispielsweise darin zu sehen, dass Impfungen vor Krankheiten schützen können. Hingegen beziehen sich die divergierenden Fakten hierbei $u$. a. auf die Abschätzung von tatsächlichen oder vermeintlichen Risiken beim und durch das Impfen im Vergleich zum Nutzen bestimmter Impfungen.

Die in diesem Modell skizzierten Vermittlungsmodi sollen die Komplexität eines transparenten Vermittlungsprozesses zwischen Experten und Laien im Diskurs und mit ihr die Herausforderungen, denen sich die linguistische Vermittlungsforschung gegenübersieht, verdeutlichen. Um die dargestellten Modi an sprachlichen Manifestationen $\mathrm{zu}$ veranschaulichen, bedarf es sowohl einer lexikalischen als auch einer grammatischen Betrachtung von Vermittlungsaussagen (geschrieben, gesprochen und multimodal).

Bei der lexikalischen Betrachtung kann ich vor allem vor dem Hintergrund meiner rechtslinguistischen Untersuchungen betonen (Felder \& Vogel 2017), wie sehr die sozio-kommunikativen Praktiken beim Dechiffrieren rechtlicher 
Termini zu beachten sind. Besonders im Recht wird deutlich, dass das Nachschlagen von Bedeutungen einschlägiger Rechtstermini nur bedingt hilft, wenn es um die angemessene Verbalisierung rechtlicher Sachverhalte geht. Dies ist zum Beispiel ein klarer Unterschied zu Wissenschaften, die als Terminologiesprachen gelten und die ihre fachlichen Praktiken terminuszentrierter bestreiten - so exemplarisch in den Fachsprachen der angewandten Wissenschaften mit ihrer (im Vergleich zur Syntax) starken Terminologisierung wie z. B. in den Ingenieurwissenschaften oder der Medizin (vgl. Roelcke 2020: 51). Adamzik formuliert zu Recht über den Zugang zum Fachsprachengebrauch über Wörterbücher:

Wenn man diese Versuche der Rekonstruktion des Sprachgebrauchs zum Ausgangspunkt der Beschreibung wählt, zäumt das Pferd beim Schwanz auf. Damit kommen wir auf die These [...] zurück, wonach die Formel Vom (Fach-)Wort zum (Fach-)Text die Entwicklung von Fach-Sprachen auf den Kopf stellt.

(Adamzik 2018: 288, Hervorhebung im Original)

- und damit auch ihren Erwerb, mag man ergänzen. Insofern ist die Lexik in manchen Disziplinen aus linguistischer Sicht ,pragmatischer' als in anderen.

Was die syntagmatische Ebene und die Verweisstrukturen in fachsprachlichen Zeichenketten angeht, möchte ich das Recht wiederum als Exempel dafür anführen, dass die Verweisstrukturen von besonderer Bedeutung sind. Dieser Aspekt zeigt sich nicht nur in den komplexen Verweisstrukturen innerhalb von Rechtsnormen, sondern auch in ihrer Weiterverarbeitung durch juristische Funktionsträger wie z. B. Anwälte, Richter, Fachjuristen in Behörden, Unternehmen usw.

Die hier vorgenommene Modellierung kann ausgedehnt werden - und zwar in weitere Modellierungen prototypischer Transformationen (,VermittlungsBlaupause`). Dabei ist zu denken an individuell empfundene oder kollektiv thematisierte Probleme und Risiken (vgl. weiterführend Müller \& Vogel 2014: 41) wie z. B. in den folgenden Bereichen: Gesundheit (Impfpolitik, GenScreening, medizinische Prognostik etc.), Ernährung (Sicherung, Nachhaltigkeit, Interessenkonflikte zwischen Ernährungsfragen und Energieversorgung mit „Biokraftstoff“), rote/grüne/weiße Biotechnologie, Synthetische Biologie, internationalisierte Wirtschaft, nationales und internationales Recht, Klimawandel, problemorientierte Technikfolgenabschätzung, Migration-Integration und sozio-kulturelle Zugehörigkeit, individuelles Ohnmachtsempfinden angesichts übermächtiger Gesellschaftsstrukturen in Gestalt ,verkrustet‘ wahrgenommener Interessens- und Lobbypolitik, psychische Überforderung und ökonomischer ,Dauerstress‘, psycho-soziale Abhängigkeitsverhältnisse durch neue 
Medien, Datenschutz in neuen Medien, Kundenprofile und Verbraucherunabhängigkeit usw. Es handelt sich hierbei nur um ein paar Beispiele, welche die Leitidee illustrieren sollen.

An diesen Exempeln wird evident: Wissensgesellschaft als Kommunikationsgesellschaft reflektiert den Umgang mit Wissen, Nicht-Wissen und Risiken und lotet die Chancen aktiver Bürgerpartizipation aus. Bovet \& Huwendiek (2014: 113) sprechen dabei von der „Erschließungsperspektive“ eines Laien.

\section{Das Gesellschaftspolitische im Deskriptiven: Fazit und Ausblick}

Die Unterscheidung zwischen Daten als Gegebenem (= nach allgemein akzeptierten Kriterien gewonnenen, oft gemessenen Größen) und Fakten (= gemachte und gedeutete Tatsachen, die bestreitbar sind) wurde oben als heuristisches Mittel zum Transparent-Machen von Streitfragen und den damit verbundenen Geltungsansprüchen von unstrittigen und streitbaren Bezugspunkten stark gemacht. Eine glaubwürdige Diskursanalyse fachlicher Gegenstände mit dem Ziel, sie angemessen zu vermitteln, muss diesen Unterschied für (relative) Laien transparent machen. Eine solche Haltung trägt zur Glaubwürdigkeit wissenschaftlichen Arbeitens bei und ist letztlich auch demokratischer Auftrag einer linguistischen, deskriptiven Forschung.

Wie politisch ist eigentlich die deskriptiv orientierte Wissenschaft bzw. die Linguistik? Diese Frage möchte ich abschließend aufgreifen - ich habe es in den einleitenden Worten kurz angesprochen. Die Frage berührt den Kern wissenschaftlichen Selbstverständnisses. Besonders häufig wird dieser Gesichtspunkt in der sprachwissenschaftlich inspirierten Diskursanalyse diskutiert, besonders dort, wo Critical Discourse Analysis (CDA) und Linguistische Diskursanalyse (LDA) ins Gespräch kommen.

Meines Erachtens zeigt sich die Politizität (ein Kunstwort für den graduell modellierten Charakter politikrelevanten Handelns) wissenschaftlichen Arbeitens zwar auch im Äußern einer Meinung, aber vielmehr oder in noch stärkerem Maße bei der Auswahl des wissenschaftlichen Gegenstandes selbst und in dem vom Wissenschaftler formulierten Erkenntnisinteresse. So ist der Nachweis, wie sich eine gesellschaftliche Ungerechtigkeit sprachlich manifestiert, ohne Zweifel sehr politisch und verdienstvoll. Nicht minder politisch - wenn auch anders gelagert - ist das Transparent-Machen sprachlich instruierter Wahrnehmungsfolien von Wissen und damit evozierter Weltbilder und Denkmuster. Schließlich 
ist es uns nur in geringem Ausmaß möglich, über Primärerfahrungen unsere individuelle Wissensformation zu speisen. Wir sind größtenteils auf die symbolvermittelte Wissensdarstellung kollektiver Wissenssysteme und der ihnen inhärenten idiomatischen Ordnung angewiesen. Die in den Medienwissenschaften von Sigfried J. Schmidt (1996) in „Die Welten der Medien“ stark gemachte Unterscheidung zwischen Wirklichkeit und Realität ist hierbei hilfreich: Unter Wirklichkeit wird die subjektive, mit den originären Sinnen erfahrbare und begreifbare Welt verstanden, Realität ist das medial konstituierte und sprachlich also zwangsläufig gestaltete Szenario davon, die sog. Medienrealität als vermittelte Welt.

Vor diesem Hintergrund der Differenzierung partizipieren Staatsbürger an der sog. Wissensgesellschaft durch kommunikative Interaktion. Als Rezipienten des sog. Informationszeitalters sollten sie sich idealiter einschalten in den Diskurs und in die dort vorgenommenen Aushandlungen. Der Zugang zur Ressource Wissen muss reflektiert und dialogisch begleitet werden. Denn als Rezipienten von Wissen sind Bürger in erheblichem Maße mit Realität konfrontiert, also mit sprachlichen Produkten, die eine perspektivierte Wissenskonstitution vorgeben. In der Rezeption von gesellschaftspolitisch relevanten Wissensbeständen haben wir es demnach mit gestalteten Materialien in sprachlicher Form zu tun, die interessengeleitete und perspektivisch instruiertes Wissen in kollektiv rezipierte (Medien-)Realität verwandelt haben. Sprach- und Bildzeichen sowie Zeichenverkettungen sind daher ein perspektivierter Ausschnitt von Welt (perspektivierte Wissenskonstitution zwecks gesellschaftlicher Einflussnahme) zur interessengeleiteten Konstitution von Realität im Spektrum verschiedener Wirklichkeiten (vgl. Felder 2013: 16).

Sprache ist das Medium für die Genese und den Transfer von Wissen. Sie fungiert dabei zugleich als Resonanzkörper komplexer Wissensbestände und der damit einhergehenden Kontroverse um den angemessenen Umgang mit Wissen. Wie Diskursakteure durch sprachliche Perspektivierungen Sachverhalte konstituieren, verknüpfen und bewerten, wurde exemplarisch an Ausschnitten des Impfdiskurses gezeigt. Die sich dabei auf der sprachlichen Oberfläche entfaltende Faktizitätsherstellung innerhalb der Diskursgemeinschaft wird an der für die Debatte paradigmatischen Kontroverse um Risiken veranschaulicht (z. B. Impfen). Wenn sich in Sprache riskantes Wissen sedimentiert, dann kann die Diskurslinguistik induktiv erschlossene Deutungsangebote liefern, indem sie Diskursverläufe systematisch $\mathrm{zu}$ erfassen und beschreiben vermag. Diese linguistische Leistung des Ans-Licht-Bringens der Faktenherstellung stellt eine Hilfe bei der Interpretation dar. Das zugrundliegende Gedankengebäude des Beitrags lautet deshalb: Strukturelle Dialogizität ist eine kommunikationstheo- 
retische und kommunikationspraktische Denkfigur mit Orientierungsfunktion für partizipative Wissensgesellschaften.

Diesem Leitgedanken folgend können unter methodologischen Gesichtspunkten Vermittlungskonzepte entwickelt werden, die neben dem sehr anspruchsvollen und idealistischen Bild vom mündigen Bürger (als Leitvokabel einem der Aufklärung verpflichteten Staatsverständnis) einen Bürger stellt, der kommunikative Praktiken zwischen Experten, Lobbyisten, Politikern und sonstigen Entscheidern einer Gesellschaft in den Grundzügen (d. h. im Hinblick auf Perspektiven, Interessen, Vernetzung usw.) zu durchschauen vermag. Das Bild vom mündigen Bürger muss um den kommunikations- und wahrnehmungsgeschulten ergänzt werden. Dies ist umso mehr angezeigt, als gerade im Internet tatsächliche und vermeintliche Daten, Fakten bis hin zu Verschwörungstheorien (die einer seriösen Recherche nicht standhalten) dargeboten werden. Eine linguistische Antwort sollte die Stärkung eines besonders kompetenten zoon politikon sein. ${ }^{4}$ Damit können kollektiv eingeschätzte Risiken wie auch individuelles Nicht-Wissen und Risikoempfinden aktiv bearbeitet werden und die Partizipationsfähigkeit der einzelnen Bürger gestärkt werden.

\section{Literaturverzeichnis}

Adamzik, Kirsten (2018): Fachsprachen. Die Konstruktion von Welten. Tübingen: A. Francke Verlag.

Antos, Gerd \& Sigurd Wichter (Hrsg.) (2005): Wissenstransfer durch Sprache als gesellschaftliches Problem. Frankfurt am Main: Peter Lang.

Assmann, Aleida (1999): Erinnerungsräume. Formen und Wandlungen des kulturellen Gedächtnisses. München: Beck.

Atayan, Vahram, Ekkehard Felder, Bettina Fetzer, Anna Mattfeldt, Daniele Moretti, Annika Straube \& Daniel Wachter (2020): Europäische Diskursgemeinschaft. Projektskizze einer sprachvergleichenden Diskursanalyse. Linguistik online 103 (3), 23-66. http://dx.doi.org/10.13092/lo.103.7113.

Becker, Andrea (2001): Populärmedizinische Vermittlungstexte. Studien zur Geschichte und Gegenwart fachexterner Vermittlungsvarietäten. Tübingen: Niemeyer.

Bittlingmayer, Uwe \& Ullrich Bauer (Hrsg.) (2006): Die „Wissensgesellschaft“: Mythos, Ideologie oder Realität? Wiesbaden: VS, Verlag für Sozialwissenschaften.

Bloching, Sven (2018): Fakten als Garant für Wahrheit? Faktizitätsherstellung in Texten am Beispiel des Faktenchecks. Bachelorarbeit - zugänglich über Prof. Felder an der Universität Heidelberg.

4 Siehe dazu https://scilogs.spektrum.de/semantische-wettkaempfe/sprachlicher-tanz-umdie-mitte-strategie-oder-irrsinn/ (letzter Zugriff 31.05.2021). 
Bock, Bettina M. \& Gerd Antos (2019): ,Öffentlichkeit‘ - ,Laien ‘ - Experten‘: Strukturwandel von ,Laien ‘ in Diskursen über ,Sprache‘. In Gerd Antos, Thomas Niehr \& Jürgen Spitzmüller (Hrsg.), Handbuch Sprache im Urteil der Öffentlichkeit, 54-79. Berlin, Boston: De Gruyter.

Bovet, Gislinde \& Volker Huwendiek (Hrsg.) (2014): Leitfaden Schulpraxis. Pädagogik und Psychologie für den Lehrberuf. 7. Aufl. Berlin: Cornelsen Scriptor.

Bromme, Rainer, Regina Jucks \& Riklef Rambow (2004): Experten-Laien-Kommunikation im Wissensmanagement. In Gabi Reinmann \& Heinz Mandl (Hrsg.), Psychologie des Wissensmanagements. Perspektiven, Theorien und Methoden, 114-126. Göttingen u. a.: Hogrefe.

Duden - Das Herkunftswörterbuch (2014): Etymologie der deutschen Sprache. Redaktionell bearbeitet von Jörg Riecke. 5. Aufl. Berlin.

Engelhardt, Anina \& Laura Kajetzke (Hrsg.) (2015): Handbuch Wissensgesellschaft. Theorien, Themen und Probleme. Bielefeld: transcript.

Felder, Ekkehard (Hrsg.) (2006): Semantische Kämpfe. Macht und Sprache in den Wissenschaften. Berlin, New York: De Gruyter.

Felder, Ekkehard (Hrsg.) (2013): Faktizitätsherstellung in Diskursen. Die Macht des Deklarativen. Berlin, Boston: De Gruyter.

Felder, Ekkehard (2015): Lexik und Grammatik der Agonalität in der linguistischen Diskursanalyse. In Heidrun Kämper \& Ingo Warnke (Hrsg.), Diskurs-interdisziplinär. Zugänge, Gegenstände, Perspektiven, 87-121. Berlin, New York: De Gruyter.

Felder, Ekkehard \& Andreas Gardt (Hrsg.) (2015-2020): Handbücher Sprachwissen (HSW). Berlin, Boston: De Gruyter.

Felder, Ekkehard \& Friedemann Vogel (Hrsg.) (2017): Handbuch Sprache im Recht. Berlin, Boston: De Gruyter.

Felder, Ekkehard (2018a): Anmaßungsvokabeln: Sprachliche Strategien der Hypertrophie oder der Jargon der Anmaßung. In Martin Wengeler \& Alexander Ziem (Hrsg.), Diskurs, Wissen, Sprache, 215-240. Berlin, Boston: De Gruyter.

Felder, Ekkehard (2018b): Wahrheit und Wissen zwischen Wirklichkeit und Konstruktion. In Ekkehard Felder \& Andreas Gardt (Hrsg.), Wirklichkeit oder Konstruktion? Sprachtheoretische und interdisziplinäre Aspekte einer brisanten Alternative, 371-398. Berlin, Boston: De Gruyter.

Habermas, Jürgen (1971): Vorbereitende Bemerkungen zu einer Theorie der kommunikativen Kompetenz. In Jürgen Habermas \& Niklas Luhmann (Hrsg.), Theorie der Gesellschaft oder Sozialtechnologie - Was leistet die Systemforschung?, 101-141. Frankfurt am Main: Suhrkamp.

Heringer, Hans Jürgen (1979): Verständlichkeit - ein genuiner Forschungsbereich der Linguistik? Zeitschrift für germanistische Linguistik 3, 255-278.

Heringer, Hans Jürgen (1984): Textverständlichkeit. Leitsätze und Leitfragen. Zeitschrift für Literaturwissenschaft und Linguistik 14 (55), 57-70.

Jacob, Katharina (2017): Linguistik des Entscheidens. Eine kommunikative Praxis in funktionalpragmatischer und diskurslinguistischer Perspektive. Berlin, Boston: De Gruyter.

Janich, Nina, Alfred Nordmann \& Liselotte Schebek (Hrsg.) (2012): Nichtwissenskommunikation in den Wissenschaften. Interdisziplinäre Zugänge. Frankfurt am Main, Berlin: De Gruyter.

Keller, Reiner (2010): Wissenssoziologische Diskursanalyse. Grundlegung eines Forschungsprogramms. 3. Aufl. Wiesbaden: Springer VS. 
Knoblauch, Hubert (1995): Kommunikationskultur. Die kommunikative Konstruktion kultureller Kontexte. Berlin, New York: De Gruyter.

Kopperschmidt, Josef (1990): Gibt es Kriterien politischer Rhetorik? Versuch einer Antwort. Diskussion Deutsch 21, 479-501.

Lyotard, Jean Francois (1987): Der Widerstreit. München: Fink.

Mattfeldt, Anna (2018): Wettstreit in der Sprache: Ein empirischer Diskursvergleich zur Agonalität im Deutschen und Englischen am Beispiel des Mensch-Natur-Verhältnisses. Berlin, Boston: De Gruyter.

Müller, Marcus \& Friedemann Vogel (2014): Risikotechnologien in europäischen Mediendiskursen: Der korpuslinguistische Zugriff am Beispiel „Biotechnologie“. In Andreas Lösch \& Marcus Müller (Hrsg.), Risikodiskurse/Diskursrisiken - Sprachliche Formierungen von Technologierisiken und ihre Folgen. Themenschwerpunkt (2/2014) der Zeitschrift „Technikfolgenabschätzung - Theorie und Praxis“, 40-48. Karlsruhe: Institut für Technikfolgenabschätzung und Systemanalyse (ITAS).

Roelcke, Thorsten (2020): Fachsprachen. 4. Aufl. Berlin: Erich Schmidt.

Schmidt, Siegfried J. (1996): Die Welten der Medien: Grundlagen und Perspektiven der Medienbeobachtung. Braunschweig, Wiesbaden: Vieweg.

Schnedermann, Theresa (i. Vorb.): Die Macht des Definierens. Eine diskurslinguistische Untersuchung zum „Burnout-Syndrom“. Berlin, Boston: De Gruyter.

Searle, John R. (1997): Die Konstruktion der gesellschaftlichen Wirklichkeit. Zur Ontologie sozialer Tatsachen. Reinbek: Rowohlt. (Originaltitel: The Construction of Social Reality. New York 1995).

Steger, Hugo (1988): Erscheinungsformen der deutschen Sprache. ,Alltagssprache‘ - ,Fachsprache'- ,Standardsprache' - ,Dialekt‘ und andere Gliederungstermini. Deutsche Sprache. Zeitschrift für Theorie, Praxis, Dokumentation 16, 289-319.

Warnke, Ingo (2009): Die sprachliche Konstituierung von geteiltem Wissen in Diskursen. In Ekkehard Felder \& Marcus Müller (Hrsg.), Wissen durch Sprache. Theorie, Praxis und Erkenntnisinteresse des Forschungsnetzwerks »Sprache und Wissen «, 113-140. Berlin, New York: De Gruyter.

Weingart, Peter, Martin Carrier \& Wolfgang Krohn (Hrsg.) (2007): Nachrichten aus der Wissensgesellschaft. Analysen zur Veränderung der Wissenschaft. Weilerswist: Velbrück Wiss.

Wichter, Sigurd \& Gerd Antos (Hrsg.) (2001): Wissenstransfer zwischen Experten und Laien. Frankfurt am Main: Peter Lang. 
